\title{
Corrigendum to: 'First ever report on the antimicrobial activities of some selected mangrove halophytes of Sundarban against milk spoilage microflora' [Braz. J. Biol. Sci. (2017), vol. 4, no. 8, p. 273-292]
}

\section{Abhishek Mukherjee*, Subhajit Das, Sabyasachi Chakraborty, Anwesa De and Tarun Kumar De}

Department of Marine Science, Ballygunge Science College. University of Calcutta. India.*Email abmsws@gmail.com.

The first author reports an error in the original version of this article. In the Figure 2 some of the names of the mangroves have been incorrectly assigned to their respective pictures. the article.

This error has now been corrected in the PDF version of

The authors would like to apologize for any inconvenience caused.
Received

January 9, 2021

Accepted

January 10, 2021

Available on line January 31, 2021

Released April 30, 2021

(2)

Open Acess Full Text Article

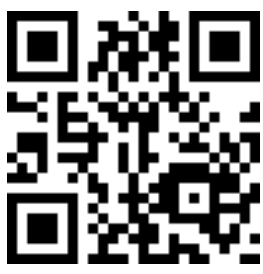

D) 0000-0001-6690-5193 Abhishek Mukherjee

(ㄷ) 0000-0002-7824-6962 Subhajit Das

D) 0000-0001-9691-4314

Sabyasachi

Chakraborty

D 0000-0002-2945-2428

Anwesa De

D 0000-0003-2733-7116

Tarun Kumar De 
The selected mangrove species

Eight true mangrove species were selected for the study (Figure 2) based on the previous studies on some of the species elsewhere in the world on their antimicrobial potentiality, while some among the selected ones have never been investigated upon before. No studies on these species against milk spoilage have been done so far.

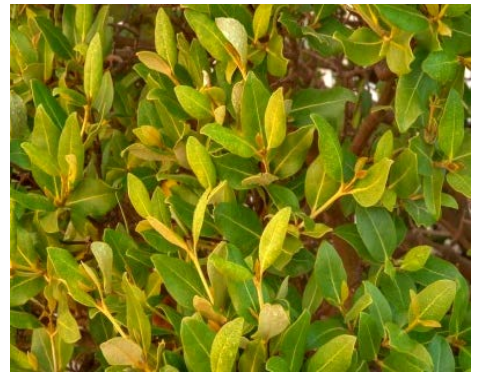

A: Avicennia marina.

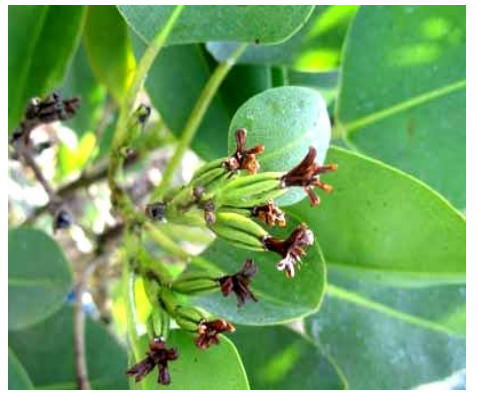

D: Aegialitis rotundifolia.

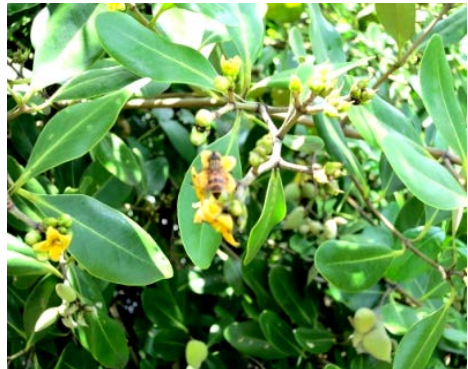

B: Avicennia officinalis.

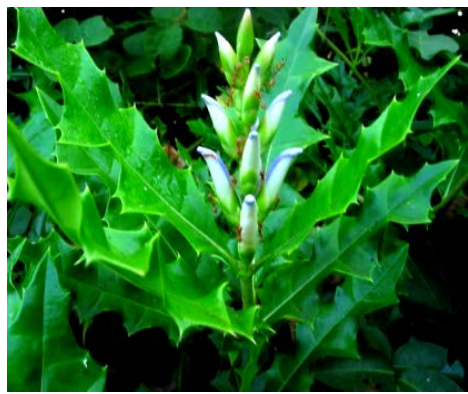

E: Acanthus ilicifolius.

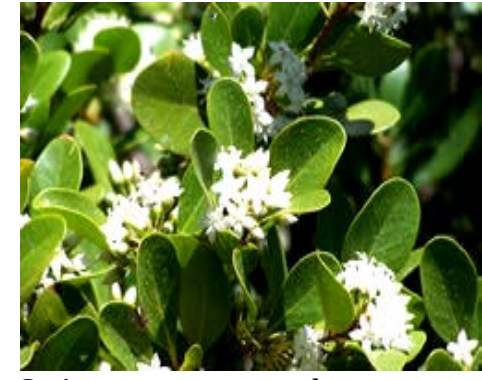

C: Aegiceras corniculatum.

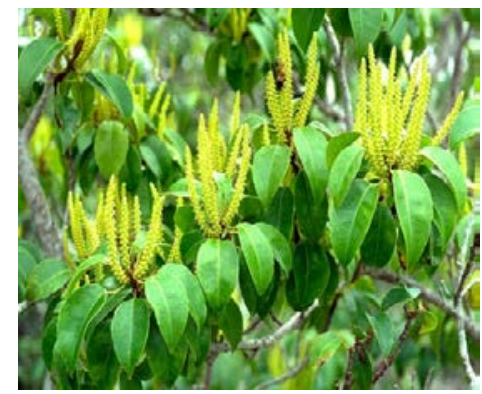

F: Excoecaria agallocha.

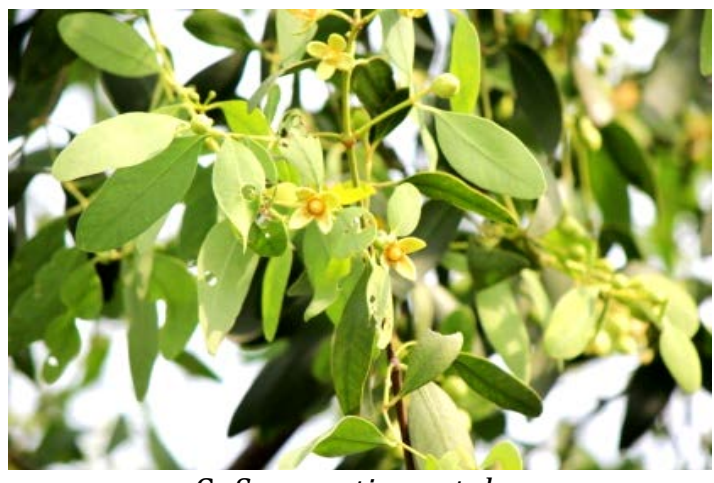

G: Sonneratia apetala.

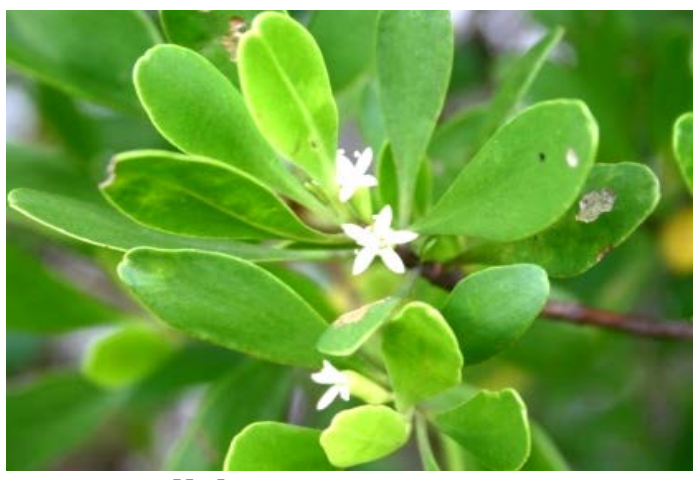

H: Lumnitzera racemosa.

Figure 2. Photographs of the selected mangrove species.

\section{Reference}

Mukherjee, A.; Das, S.; Chakraborty, S.; De, A.; De, T. K. First ever report on the antimicrobial activities of some selected mangrove halophytes of Sundarban against milk spoilage microflora. Brazilian Journal of Biological Sciences, v. 4, no. 8, p. 273-292, 2017. https://doi.org/10.21472/bjbs.040806 